\section{soluo mental}

Ruiz Torres, Gabriela Mariana; Medina-Mora Icaza, María Elena La percepción de los adolescentes sobre el consumo de alcohol y su relación con la exposición a la oportunidad y la tentación al consumo de alcohol

Salud Mental, vol. 37, núm. 1, enero-febrero, 2014, pp. 1-8

Instituto Nacional de Psiquiatría Ramón de la Fuente Muñiz

Distrito Federal, México
Salud Mental

perezrh@imp.edu.mx

Instituto Nacional de Psiquiatría Ramón de la

Fuente Muñiz

México
ISSN: 0185-3325

Disponible en: http://www.redalyc.org/articulo.oa?id=58229962001

- Cómo citar el artículo

- Número completo

- Más información del artículo

- Página de la revista en redalyc.org 


\title{
La percepción de los adolescentes sobre el consumo de alcohol y su relación con la exposición a la oportunidad y la tentación al consumo de alcohol
}

\author{
Gabriela Mariana Ruiz Torres, ${ }^{1}$ María Elena Medina-Mora Icaza²
}

Artículo original

\section{SUMMARY}

\section{Introduction}

Alcohol is an endemic problem in our country. It affects mainly adolescents, and is associated with the availability and individual characteristics and context that favor the likelihood of experiencing, continue to use and move toward the problematic use and dependence. Substance use is also associated with being exposed to them and making decisions about their conduct towards the opportunity, so prevention may be more effective when you understand the mechanisms between the availability and behavior and exposure to opportunity and the temptation to consume. This work addresses these constructs in a group of adolescents using a qualitative approach and explores the perception of the constructs, their association with the use and the strategies used by adolescents to avoid consumption.

\section{Methodology}

The sample was composed by 60 adolescents, 30 experimentalists and 30 alcohol abusers, divided into six groups.

\section{Results}

The results show a clear identification and definition of constructs exposed to the opportunity and temptation to consume. Family parties and invitations are considered situations associated with exposure to the opportunity, and temptation consumption is associated with unpleasant emotions. In the coping strategies to avoid drinking, experimenters think about the consequences of consumption, in saying no, and in respecting the rules and decisions, while the abusers think of the fun and never refuse an invitation from a friend.

\section{Discussion}

Results are proposed for inclusion in models of prevention and care for specific populations.

Key words: Adolescents, exposure to opportunity, temptation to consume, alcohol consumption.

\section{RESUMEN}

El consumo de alcohol es un problema endémico en nuestro país. Afecta principalmente a los adolescentes y se asocia con la disponibilidad y con características individuales y del contexto que favorecen la probabilidad de experimentar, continuar usando y avanzar hacia el consumo problemático y la dependencia. El uso de sustancias también se relaciona con estar expuesto a ellas y tomar decisiones sobre su conducta frente a la oportunidad. Así, la prevención puede ser más efectiva cuando se comprenden los mecanismos intermedios entre la disponibilidad y la conducta como la exposición a la oportunidad y la tentación al consumo. Este trabajo aborda estos constructos en un grupo de adolescentes mediante una aproximación cualitativa y explora la percepción de los constructos, su asociación con el consumo y las estrategias que utilizan los adolescentes para evitar el consumo.

\section{Método}

La muestra se integró con 60 adolescentes, 30 experimentadores y 30 abusadores de alcohol, divididos en seis grupos. Los resultados muestran una clara identificación y definición de los constructos exposición a la oportunidad y tentación al consumo. Fiestas familiares e invitaciones son consideradas situaciones asociadas con la exposición a la oportunidad; y la tentación al consumo se asocia con emociones desagradables. En las estrategias de enfrentamiento para evitar el consumo, los experimentadores piensan en las consecuencias del consumo, en decir no y en respetar las reglas y decisiones; en tanto que los abusadores piensan en la diversión y nunca rechazarían la invitación de un amigo.

\section{Discusión}

A partir de los resultados se hacen propuestas para su incorporación en modelos de prevención y atención para poblaciones específicas.

Palabras clave: Adolescentes, exposición a la oportunidad, tentación por el consumo, consumo de alcohol.

Facultad de Psicología. Universidad Nacional Autónoma de México.

2 Dirección General del Instituto Nacional de Psiquiatría Ramón de la Fuente Muñiz.

Correspondencia: Mtra. Gabriela Mariana Ruiz Torres. Capulín 228, Edif A7, Depto 401, Arcos del Sur, Xochimilco, 16010, México, D.F. Teléfonos: (55) 6277 -6437 y (55) 5850-5952. Celular: 55 1800-6521. E-mail: gabrielamarianaruiztorres@hotmail.com; gabitaruiz26@gmail.com

Recibido: 29 de enero de 2013. Aceptado: 31 de octubre de 2013. 


\section{INTRODUCCIÓN}

En México, el consumo de alcohol es considerado como un problema de salud pública que afecta a diferentes segmentos de la población. En el último reporte de la Encuesta Nacional de Adicciones $2008^{1}$ se muestra que los adolescentes son el sector más vulnerable debido a que en esta etapa existen constantes cambios en todas las áreas de su vida, así como su involucramiento en conductas poco saludables y en ocasiones problemáticas como el consumo de sustancias.

La Encuesta de Consumo de Drogas en Estudiantes, en su medición del 2006, ${ }^{2}$ un incremento en el consumo tanto de sustancias legales como ilegales. Para el caso de las drogas ilegales (mariguana, cocaína, inhalables, tranquilizantes y otras drogas de carácter médico usadas sin prescripción), se observa que para el año 2003 el consumo en jóvenes representó el 15.2\%, en tanto que para la medición de 2006, el $17.8 \%$ de los jóvenes había consumido drogas. Por lo que respecta al consumo de sustancias legales (alcohol y tabaco) se presentó un incremento en cuanto al consumo de alcohol como droga de inicio (de una a tres copas por ocasión, menos de una vez al mes) y como una droga de abuso (consumo mayor a cinco copas de alcohol por ocasión), situación que afecta al 25.2\% de la población joven. Adicionalmente estos resultados reflejan que la alta tolerancia social y la baja percepción de riesgo asociada al abuso de alcohol se relacionan con el incremento del consumo de otras sustancias que acarrean una serie de consecuencias a la salud de los adolescentes.

Es importante mencionar que el consumo de sustancias se asocia con algunas características de la adolescencia, como la búsqueda de identidad, la autonomía e independencia; ${ }^{3-7}$ así como con la adopción de roles de adulto ${ }^{8}$ y la concepción de inmunidad frente a los riesgos, situación que favorece la búsqueda de sensaciones novedosas momentáneas e inmediatas. ${ }^{9,10}$

Ante la búsqueda de estas sensaciones novedosas, los adolescentes en ocasiones se involucran en conductas de diferente nivel de riesgo, siendo las de alto riesgo las de mayor preocupación. Dentro de las conductas de riesgo a las cuales se enfrenta el adolescente se encuentran el inicio en la vida sexual sin protección, la adopción de estilos de vida poco saludables, el inicio en el consumo de sustancias a edades mucho más tempranas y el abuso de alcohol, tabaco y otras drogas. ${ }^{11}$

El consumo de sustancias no es una problemática de salud pública que se presente de un día para otro, pues está íntimamente relacionada con diversos factores de riesgo que se definen como todas aquellas situaciones en las cuales es más probable que se presente una conducta. ${ }^{12}$ Entre los factores de riesgo más cercanos al consumo de sustancias se encuentran el entorno social, la baja percepción de riesgo, la alta tolerancia del contexto hacia el consumo, la exposición a la oportunidad y la tentación al consumo; así como algunos eventos estresantes de la vida. ${ }^{13,14}$
Algunos autores mencionan que la exposición a la oportunidad y la tentación al consumo son condiciones necesarias para que el individuo avance por una trayectoria riesgosa de consumo, la cual se inicia con la experimentación y puede alcanzar niveles de dependencia. ${ }^{15}$

Esta situación se explica de manera inicial porque el individuo se encuentra ante una extensa y constante exposición a la oportunidad, definida como cualquier situación en la que el individuo se encuentra ante la invitación o el ofrecimiento de consumir una sustancia. Otro de los elementos que complementa esta trayectoria de consumo en el individuo corresponde a la experiencia previa asociada con las expectativas de los resultados, dando lugar a la búsqueda de situaciones en las que es posible que se presente el consumo, lo que puede ser denominado como tentación al consumo. Sin embargo, es necesario comentar que el individuo se encuentra en una constante disyuntiva entre la exposición a la oportunidad, el interés por consumir y la conciencia de las consecuencias que acarrea consigo dicho consumo. ${ }^{16-18}$

Estudios orientados a comprender las principales circunstancias por las cuales pudiera presentarse una oportunidad de consumo muestran que una disminución en la supervisión de los niños y adolescentes tiene una relación causal con la progresión entre la primera oportunidad de consumo y la primera ocasión en que busca el consumo por su propio interés, ya sea de alcohol, tabaco, mariguana o inhalables. ${ }^{19}$

El concepto de "Exposición a la oportunidad" en ocasiones resulta poco claro. Una explicación inicial corresponde al principio epidemiológico de exposición a enfermedades infecciosas propuesto por Maxcy en 1943 y el cual es adaptado al fenómeno de las adicciones. Así, identifican que la progresión en el consumo de sustancias se relaciona con la posibilidad de estar frente a una sustancia, por lo que argumentan que aquellos adolescentes que han consumido alcohol y tabaco tienen una mayor probabilidad para involucrarse en el consumo de mariguana; en tanto que a aquellas personas que experimentaron con mariguana les resulta más sencillo involucrarse con el consumo de drogas fuertes (cocaína, metanfetaminas, etc.). ${ }^{20}$

Estudios más recientes ${ }^{14}$ destacan que la exposición a la oportunidad debe ser entendida como la posibilidad de haber consumido alcohol ya sea por la invitación de cualquier persona, por haber buscado la ocasión para hacerlo, o bien por encontrarse en alguna circunstancia que propiciara el consumo sin buscarla intencionalmente. En este sentido, otra de las condiciones necesarias para involucrarse en el consumo de alcohol, tabaco y otras drogas es la tentación al consumo, la cual ha sido estudiada como un predictor en el mantenimiento de la abstinencia de una persona exconsumidora con indicadores de dependencia a una sustancia en particular y como un factor de predicción ante una recaída, que lo lleva a utilizar estrategias de enfrentamiento para evitar el consumo. 
Por otro lado, en la trayectoria del uso al consumo, la tentación se centra en la intención para buscarlo, donde se involucra la utilización de habilidades para rehusarse o resistirse. Por lo anterior, la tentación debe entenderse como la posibilidad de consumir alcohol u otras sustancias, siempre y cuando el individuo se encuentre frente a la oportunidad, tenga la intención y el deseo por consumir. Otra de las explicaciones de la tentación involucra el estar de acuerdo e interesado en el consumo de alcohol u otras drogas. ${ }^{21}$

Cabe destacar que el consumo de alcohol y el uso de drogas se relacionan con la utilización de las estrategias de enfrentamiento a la tentación, debido a que no todos los esfuerzos por resolver una situación de presión al consumo resultan efectivos, por lo que lo favorecen hasta llegar al abuso.

Así, otro de los factores relacionados con el inicio, el mantenimiento, la reducción y la abstinencia del consumo de sustancias es la utilización de estrategias de enfrentamiento por parte de los individuos ante situaciones específicas. ${ }^{16}$ Las estrategias de enfrentamiento son definidas como los esfuerzos concretos de una persona para dar respuesta o solución a una situación específica. ${ }^{22}$

En el ámbito de las adicciones, los individuos que se involucran en el consumo de alcohol muestran estrategias de enfrentamiento que reflejan un control momentáneo de la situación, es decir, la evitan, huyen del evento, aceptan una copa de alcohol sólo para evitar la insistencia de otros, etc. En tanto que aquellos individuos que muestran estrategias de enfrentamiento orientadas al control, manejo y cambio de la situación logran la solución de problemas, consideran las consecuencias de su consumo, toman decisiones, plantean respuestas asertivas y rechazan una copa de alcohol, lo que disminuye la probabilidad de involucrarse en su abuso. ${ }^{23}$

Al considerar algunos de los factores relacionados con el inicio, el mantenimiento, la reducción y la abstinencia del consumo de alcohol, se han planteado diversos modelos de atención que parten de la identificación de las causas que favorecen el uso y abuso del alcohol y sus consecuencias. Sin embargo, resulta necesaria la identificación de las causas que llevan al inicio en el consumo y que favorecen una progresión en los diferentes estados de la trayectoria de adicción, así como en el consumo de diferentes sustancias.

Una forma de identificar estas causas es por medio del desarrollo de modelos de atención enfocados a variables intermedias del proceso de adicción y que pueden ser más cercanas al individuo, como la exposición a la oportunidad de consumo, la disponibilidad, la tentación y las estrategias de enfrentamiento, que pueden ser una puerta de entrada para encontrar una posible explicación de las razones por las cuales el individuo inicia el consumo de alcohol y continúa o no en la carrera hacia el abuso y la dependencia a éste o a su experimentación con otras sustancias.

Así, para el desarrollo de estos modelos se requieren diversos elementos, como la identificación de las variables mediadoras, su definición y la manera en que las concep- tualiza la población a la cual se pretende dirigir la prevención, así como identificar las situaciones asociadas con estas variables y la forma en que los individuos dan solución a situaciones específicas relacionadas con la exposición a la oportunidad y la tentación al consumo.

Es por ello que el presente trabajo tiene por objetivos: inicialmente, averiguar si entre la población adolescente existe un claro entendimiento del significado de la exposición a la oportunidad y la tentación al consumo. En un segundo momento averiguar las diferencias en las circunstancias relacionadas con la exposición a la oportunidad y en las situaciones relacionadas con la tentación al consumo. Por último, la identificación de estrategias de enfrentamiento que ponen en práctica los adolescentes como una forma de tomar decisiones ante el consumo de alcohol.

\section{MÉTODO}

Los datos para la presente investigación provienen de un estudio basado en metodología cualitativa, empleando como técnica de investigación los grupos focales, que favorecen la exploración de nuevas líneas de investigación y donde se pretende identificar información asociada a conocimientos, actitudes y sentimientos de una muestra en particular. ${ }^{24}$

\section{Participantes}

En este estudio participaron seis grupos de 10 adolescentes cada uno, entre 13 y 16 años de edad. Tres grupos (30 adolescentes) se caracterizaron por estar expuestos al riesgo para el consumo de alcohol o ser experimentadores, es decir, no bebían $(46.7 \%)$ o bebían de una a tres copas estándar por ocasión de consumo (53.3\%), menos de una vez al mes y no reportaban problemas relacionados con el consumo de alcohol; la edad promedio de la mayoría (43.3\%) fue de 13 años. Con lo que respecta al género, 52.2\% fueron mujeres y $47.8 \%$ fueron hombres.

Los otros tres grupos (30 adolescentes) se caracterizaban por ser consumidores de alcohol. El 66.6\% de éstos consumía cinco o más copas estándar por ocasión de consumo hasta llegar a ocho o nueve copas; cabe mencionar que $33.4 \%$ de los participantes bebía cuatro copas por ocasión, por lo menos una vez cada quince días. El 45.7\% de los adolescentes de este grupo reportó haber tenido problemas relacionados con su forma de beber en la escuela, con la familia y con amigos. Con lo que respecta a la edad que tenían al momento del estudio, la mayoría (54.3\%) reportó 15 años. En cuanto al género, $63.4 \%$ fueron hombres y $36.6 \%$, mujeres.

\section{Instrumentos}

Para identificar a los participantes, se utilizaron diversos cuestionarios: 1. entrevista de patrón de consumo, con la que 
se evaluó el consumo de alcohol, la cantidad y la frecuencia a partir de tres preguntas: ¿Cada cuándo consumes bebidas alcohólicas?, Cuando bebes, ¿cuántas copas tomas en un mismo día o reunión?, ¿qué tan frecuentemente tomas cinco o más copas en un mismo día o reunión?; 2. un cuestionario de tamizaje de los problemas en el adolescente (POSIT) ${ }^{25}$ en su sección de adicciones, constituido por 19 preguntas, de las cuales sólo se retomaron siete (¿Has tenido dificultades porque consumes bebidas alcohólicas en la escuela?, ¿Se aburren tus amigos en las fiestas donde no sirven bebidas alcohólicas?, ¿Llevan tus amigos bebidas alcohólicas a las fiestas?, ¿Te vas a veces de las fiestas porque no hay en ellas bebidas alcohólicas?, ¿Sientes un antojo por consumir bebidas alcohólicas?, ¿Pierdes días de clase o llegas tarde a la escuela por haber consumido bebidas alcohólicas?, ¿Has sentido que no puedes controlar el antojo de consumir bebidas alcohólicas?) cuyas opciones de respuesta fueron: SÍ y NO; 3. una guía temática que orientó la discusión en los grupos focales, la cual se desarrolló ex profeso para esta investigación y estuvo constituida por cuatro áreas: consumo de alcohol, exposición a la oportunidad, tentación al consumo $\mathrm{y}$, por último, el enfrentamiento asociado con la percepción de su efectividad.

\section{Procedimiento}

Dentro de las actividades de difusión del Centro de Prevención y Atención de Adicciones de la Facultad de Psicología de la UNAM, se impartieron una serie de pláticas relacionadas con la prevención en el consumo de sustancias en dos secundarias públicas del sur de la ciudad, pertenecientes a la Secretaría de Educación Pública. Como un trabajo complementario y de apoyo al área de orientación de la escuela, se solicitó la participación voluntaria de los adolescentes en un taller de discusión. Inicialmente se identificó a los adolescentes experimentadores o que estuvieron expuestos al riesgo y en un segundo momento se identificó a los abusadores. La identificación de los participantes se llevó a cabo mediante la Entrevista de Patrón de Consumo y el POSIT. Posteriormente, se formaron seis grupos de diez adolescentes, tres de los cuales estuvieron constituidos sólo por los experimentadores y los tres restantes por abusadores.

Después de la formación de los grupos, se llevó a cabo la discusión dentro de los grupos focales, siguiendo la guía temática. Esta discusión se llevó a cabo en el lapso de una hora y media; la información fue recabada por medio de una audiograbadora. Para mantener el anonimato de los adolescentes, se emplearon seudónimos en la transcripción. Adicionalmente, durante la discusión en el grupo, se incluyó a un observador, quien se encargó de tomar nota acerca del orden y participación de cada uno de los adolescentes, además de incluir una descripción de la actitud de los mismos.

Posterior a la discusión en grupo, se transcribió la información obtenida a fin de iniciar el análisis de la misma. Para el análisis de la información se establecieron de tres a cuatro categorías para clasificar las respuestas de los adolescentes, conforme a la guía de discusión.

\section{RESULTADOS}

En el área de consumo de alcohol se observa que la mayoría de los adolescentes experimentadores (83\%) identifican que el consumo de bebidas alcohólicas es una forma de dañar el organismo y una manera de perder el control, en tanto que el $17 \%$ restante piensa que no es un problema mientras exista un consumo moderado. Por otro lado, para los adolescentes que abusan del alcohol, $60 \%$ consideran que el consumo es una forma de diversión y una manera de solucionar problemas, en tanto que $40 \%$ lo consideran como una posibilidad de obtener sensaciones placenteras.

En cuanto a la percepción de los adolescentes con respecto al No consumo de alcohol, los resultados del grupo de experimentadores muestran que $100 \%$ consideran que es una forma de cuidarse, sin dañar el organismo y que pueden divertirse sin alcohol. Por lo que respecta a los abusadores, $50 \%$ de éstos consideran que el no consumo no existe, ya que en realidad todos los adolescentes beben una gran variedad de bebidas alcohólicas y en cantidades fuertes. Sin embargo, el 50\% restante menciona que el no consumo sólo se da en algunos adolescentes que no muestran un interés por divertirse.

Entre las principales razones por las cuales se consume alcohol, se observa que $70 \%$ de los adolescentes experimentadores perciben que es por curiosidad, en tanto que el $30 \%$ restante piensa que la razón más importante por la cual se presenta este consumo es por llamar la atención y por formar parte de un grupo de amigos en particular. Por el contrario, $60 \%$ del grupo de los abusadores consideran que las razones por las cuales se bebe alcohol se relacionan con el liderazgo en un grupo, por imitación, en tanto que $30 \%$ menciona que el consumo de alcohol es por resolver problemas en su vida y el 10\% restante menciona que el consumo es por gusto.

El propósito inicial de este trabajo se centró en la definición de la "exposición a la oportunidad" y "la tentación al consumo". En el cuadro 1 se observa el porcentaje de acuerdo entre los adolescentes con respecto a las categorías que definen la exposición a la oportunidad y la tentación al consumo. Así, para el grupo de los experimentadores, la exposición a la oportunidad fue definida como una invitación al consumo, donde el alcohol está disponible y existe una tolerancia hacia él. En tanto que para el grupo de los que abusan, la categoría que definió la exposición a la oportunidad es la borrachera, seguida de la invitación, el ofrecimiento y la disponibilidad del alcohol. Por lo que respecta a la tentación al consumo, la mayoría de los experimentadores definen la tentación al consumo como un antojo de la bebida 
Cuadro 1. Porcentaje de acuerdo entre los adolescentes con respecto a las categorías que definen el concepto de exposición a la oportunidad y tentación al consumo

\begin{tabular}{|c|c|c|c|c|}
\hline \multicolumn{5}{|c|}{ Categorías y porcentajes } \\
\hline Pregunta & Experimentadores & $\%$ & Abusadores & $\%$ \\
\hline $\begin{array}{l}\text { Para ustedes, que sig- } \\
\text { nifica la siguiente fra- } \\
\text { se "estar expuesto a } \\
\text { una oportunidad de } \\
\text { consumo de alcohol" }\end{array}$ & $\begin{array}{l}\text { - Invitación } \\
\text { - Que el alcohol esté dis- } \\
\text { ponible } \\
\text { - Ofrecimiento } \\
\text { - Tolerar el consumo de } \\
\text { otras personas }\end{array}$ & $\begin{array}{l}35 \\
25 \\
20 \\
20\end{array}$ & $\begin{array}{l}\text { - Invitación } \\
\text { - Que el alcohol esté disponible } \\
\text { - Ofrecimiento } \\
\text { - Fiesta segura } \\
\text { - Borrachera } \\
\text { - Compartir el momento con } \\
\text { amigos }\end{array}$ & $\begin{array}{l}25 \\
15 \\
15 \\
10 \\
25 \\
10\end{array}$ \\
\hline $\begin{array}{l}\text { Qué entienden por } \\
\text { tentación al consumo }\end{array}$ & $\begin{array}{l}\text { - Antojo } \\
\text { - Presión al consumo } \\
\text { - Ganas de consumir } \\
\text { - Ganas de sentir el efecto }\end{array}$ & $\begin{array}{l}40 \\
15 \\
30\end{array}$ & $\begin{array}{l}\text { - Ganas de consumir } \\
\text { - Antojo }\end{array}$ & $\begin{array}{l}60 \\
40 \\
15\end{array}$ \\
\hline
\end{tabular}

y las ganas por consumir, en tanto que para los abusadores el porcentaje de acuerdo se situó en las categorías de interés y las ganas por consumir.

En el cuadro 1 se muestra el porcentaje de acuerdo entre los adolescentes con respecto a las categorías que definen el concepto de exposición a la oportunidad y tentación al consumo.

Un segundo propósito de este trabajo fue la identificación de situaciones relacionadas con estos dos constructos (la "exposición a la oportunidad" y la "tentación al consumo"). En la figura 1 se muestra que $66 \%$ de los adolescentes experimentadores mencionaron que la principal situación de "exposición a la oportunidad" ocurre en reuniones familiares, en tanto que el $44 \%$ restante describe que la principal situación de exposición son las fiestas con amigos. Por su parte, $80 \%$ de los adolescentes que abusan del alcohol mencionaron que las situaciones de exposición a la oportunidad son los eventos deportivos, festejos, en tanto que el $20 \%$ restante considera que las invitaciones y eventos recreativos son situaciones de exposición.

Por lo que respecta a las situaciones relacionadas con la "tentación al consumo", la figura 2 muestra que 55\% del grupo de experimentadores y $40 \%$ de los abusadores piensan que la situación más importante está relacionada con las emociones

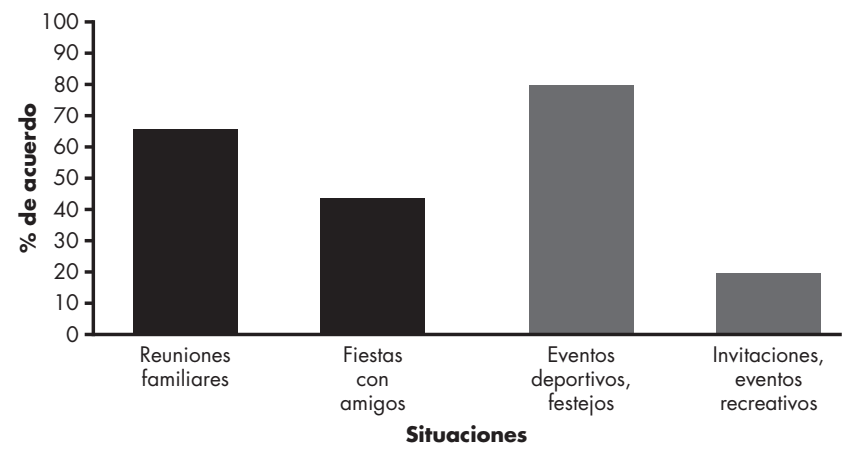

Figura 1. Percepción de las principales situaciones relacionadas con la exposición a la oportunidad. desagradables (tristeza, depresión, soledad, rechazo, presión, culpabilidad, etc.). Asímismo, 20\% de los experimentadores y $10 \%$ de los abusadores lo asociaron con la presencia de problemas familiares, en tanto que $25 \%$ de los experimentadores y $60 \%$ de los abusadores mencionaron que otra situación asociada a la tentación se vincula con momentos agradables tanto individualmente como con otras personas.

El último propósito de este trabajo se centra en la identificación de las estrategias de enfrentamiento para tomar la decisión entre beber o no ante la exposición a la oportunidad y la tentación al consumo. En este sentido, en el cuadro 2 se contemplaron estrategias para tomar la decisión entre beber o no beber, lo que se hace para evitar el consumo y lo que se hace ante el ofrecimiento de una copa. En las estrategias relacionadas con la decisión de beber una copa de alcohol o no, los resultados muestran que la mayoría (90\%) de los adolescentes experimentadores consideran que antes de que un adolescente decida beber, éste piensa en las consecuencias de sus actos, así como en la situación en la que se presenta el consumo; el 10\% restante no sabe lo que haría. En tanto que para los adolescentes abusadores, $80 \%$ de éstos mencionaron que, antes de tomar la decisión de beber, es importante pensar en la diversión y en las cosas positivas que suceden después del consumo (por ejemplo, olvidarte de los proble-

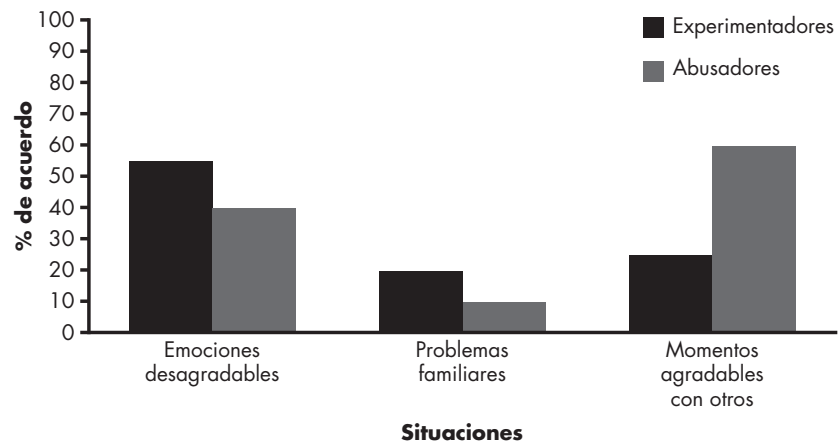

Figura 2. Percepción de las principales situaciones relacionadas con la tentación al consumo. 
mas, sentirse parte del grupo y agradar a los demás) y 20\% consideran que sólo hay que pensar en beber.

Dentro de las estrategias para evitar el consumo, la mayoría de los adolescentes que integraron el grupo de los experimentadores (53\%) plantearon como alternativas para evitar el consumo la planeación de juegos donde no se involucren bebidas alcohólicas y respetar las reglas del lugar que pudiera ser considerado como una exposición a la oportunidad (fiesta, reunión de amigos, etc.). Cabe mencionar que $47 \%$ de los adolescentes restantes mencionaron que la forma de evitar el consumo se relaciona con que el adolescente respete su decisión de no beber y que siempre diga que no, así como la búsqueda de nuevas alternativas de diversión. En el caso de los adolescentes del grupo de abusadores, 90\% de éstos comentaron que evitar el consumo es complicado, ya que "no hay fiesta sin alcohol".

Ante el ofrecimiento de una copa de alcohol, 70\% de los adolescentes del grupo de experimentadores mencionaron que decir "no" o "no me gusta el alcohol" son estrategias efectivas. El 30\% restante mencionó como estrategias decirle a quien les ofrece que "no está bien lo que hace", irse del lugar y evitar a toda costa que esa persona se les acerque. En cambio, 60\% de los adolescentes del grupo de abusadores indicaron que no rechazarían el ofrecimiento de un amigo, por lo que aceptan la copa y beben junto con éste.

En el cuadro 2 se muestra el porcentaje de acuerdo entre los adolescentes con respecto a la percepción de las posibles estrategias de enfrentamiento que usaría un adolescente ante la exposición a la oportunidad de consumo.

\section{CONCLUSIONES Y DISCUSIÓN}

El consumo de alcohol en nuestro país representa un problema de salud que va en aumento y afecta a gran parte de nuestra población, siendo los adolescentes y las mujeres el grupo más vulnerable. Hasta el momento se han planteado diversas explicaciones con respecto al inicio y mantenimiento en el consumo de alcohol. Una de las explicaciones más importantes para este trabajo es la propuesta por la Teoría Cognitiva Social, en la que se menciona que el consumo de alcohol es parte integral del desarrollo psicosocial y de la socialización en una cultura. ${ }^{26-29}$ Así, el consumo de bebidas alcohólicas en los jóvenes se da por la influencia social de la cultura, la familia y los compañeros. ${ }^{30}$

Desde la percepción de los grupos experimentadores con los cuales se llevó a cabo este estudio, hay dos concepciones con respecto al consumo de alcohol. Una está íntimamente relacionada con la socialización y lo consideraron como un elemento para compartir con otras personas siempre y cuando éste se consuma con moderación, es decir, de una a dos copas por ocasión de consumo. Una segunda concepción al respecto es que el consumo es considerado como una forma poco saludable de pasar el tiempo que en muchas ocasiones los lleva a perder el control de su comportamiento $\mathrm{y}$ a tener consecuencias asociadas con su consumo. Sin embargo, desde la percepción de adolescentes que han consumido alcohol y se han involucrado hasta el abuso, su consumo está relacionado con la concepción del alcohol como un socializador y como un agente de diversión.

Los hallazgos de este trabajo muestran que las razones más importantes por las que un adolescente puede involucrarse en el consumo son la curiosidad y el ser parte de un grupo. Sin embargo, es importante mencionar que una razón secundaria para el consumo es la solución de problemas. Estos hallazgos se relacionan con lo propuesto por Villatoro (1999), quien describe que el involucramiento en el consumo de alcohol, tabaco y otras drogas se relaciona con diversos factores de riesgo y múltiples razones, como la curiosidad, la búsqueda de identidad, la pertenencia a un grupo, la tolerancia al consumo y tener un grupo de amigos que consumen. ${ }^{11}$

El objetivo por el cual se desarrolló este trabajo se centró en averiguar si los adolescentes comprendían los constructos de "exposición a la oportunidad" y "tentación al consumo"; a partir de la revisión de los resultados de los grupos focales, se observó que los adolescentes entienden y diferencian ambos términos. Los diversos estudios ${ }^{14,20}$ muestran que la exposición a la oportunidad está relacionada con las posibilidades para el consumo sin que exista una intención por él. Para los adolescentes que participaron en este trabajo, hay una clara identificación de las situaciones en las cuales se puede estar expuesto a una oportunidad, siendo la más importante la invitación al consumo por parte de cualquier persona, así como situaciones en las que se encuentre disponible el alcohol.

\section{Cuadro 2}

\begin{tabular}{|c|c|c|c|c|c|c|}
\hline Grupo & $\begin{array}{c}\text { Tomar la decisión } \\
\text { entre beber y no beber }\end{array}$ & $\%$ & Evitar el consumo & $\%$ & Ofrecimiento de una copa & $\%$ \\
\hline Experimentadores & $\begin{array}{l}\text { - Pensar en las conse- } \\
\text { cuencias } \\
\text { - No sé lo que debo } \\
\text { hacer }\end{array}$ & $\begin{array}{l}90 \\
10\end{array}$ & $\begin{array}{l}\text { - Planear juegos sin alco- } \\
\text { hol y respetar reglas } \\
\text { - Respetar la decisión de } \\
\text { no beber }\end{array}$ & 53 & $\begin{array}{l}\text { - Decir No o No me gus- } \\
\text { ta el alcohol } \\
\text { - No está bien lo que ha- } \\
\text { ces e irse del lugar }\end{array}$ & 70 \\
\hline Abusan del alcohol & $\begin{array}{l}\text { - Diversión y conse- } \\
\text { cuencias positivas } \\
\text { - Solo hay que beber }\end{array}$ & $\begin{array}{l}80 \\
20\end{array}$ & $\begin{array}{l}\text { - Evitar el consumo es } \\
\text { complejo } \\
\text { - No sé lo que debo hacer }\end{array}$ & $\begin{array}{l}90 \\
20\end{array}$ & $\begin{array}{l}\text { - No rechazaría una } \\
\text { copa y bebería junto } \\
\text { con mi amigo }\end{array}$ & 60 \\
\hline
\end{tabular}


En cuanto a la definición de "la tentación al consumo", la información obtenida por los adolescentes reflejó que sí entienden a lo que se refiere la tentación al consumo, y enfatizan que es muy importante que se presenten dos factores para que se pueda dar el consumo de alcohol. Por un lado está el interés por consumir y, por otro, el interés por sentir los efectos del alcohol sobre el organismo. Esta información complementa lo propuesto por otros estudios, en los que se destaca la importancia de que esté presente el interés para el consumo y una oportunidad para consumir. ${ }^{16}$

La investigación de Reboussin y Anthony (2001) ${ }^{19}$ destaca que una de las situaciones asociadas con la progresión entre la exposición a la oportunidad y el primer consumo de alcohol es la disminución de la supervisión familiar. Estos hallazgos se relacionan con lo concluido por los adolescentes, quienes encontraron que una de las primeras oportunidades para el consumo es con la familia. Sin embargo, las oportunidades se incrementan en la medida en que existen fiestas con amigos en las que disminuye la supervisión por parte de personas adultas o donde hay una tolerancia mayor por el consumo de bebidas alcohólicas. Caso contrario lo representan las situaciones relacionadas con la tentación al consumo, puesto que la mayoría de las situaciones identificadas por parte de los adolescentes se vinculan con las estrategias de enfrentamiento, que son consideradas como un factor de inicio en el consumo, puesto que aquellos adolescentes que se involucran en el consumo emplean el alcohol como una estrategia de aliviar emociones desagradables y lo relacionan con la búsqueda de emociones y sensaciones de bienestar, lo cual concuerda con los hallazgos de Wills y Hirky (1996). ${ }^{23}$

Un tercer objetivo de este trabajo fue la identificación de las estrategias de enfrentamiento que los adolescentes ponen en práctica para tomar la decisión entre beber o no beber. Cabe mencionar que los adolescentes concluyeron que no en todas las ocasiones es necesario beber, por lo que aquellos que deciden no beber basan su decisión en las consecuencias negativas de sus actos bajo los efectos del alcohol. A su vez, los adolescentes que toman la decisión de beber o continuar bebiendo se basan en las consecuencias positivas del consumo. De acuerdo con la Teoría Cognitivo Social, la experiencia directa con el alcohol se vuelve importante a medida que continúa el desarrollo y la experimentación con la sustancia, ya que se refuerzan positivamente por las propiedades eufóricas que se dan en las interacciones sociales y se asocian con la anticipación de los resultados positivos.

En cuanto a las estrategias específicas para enfrentar el consumo de alcohol, los adolescentes destacaron tres estrategias importantes. La primera se relaciona con la planeación de juegos donde no se involucren bebidas alcohólicas (manejando y controlando la situación). La segunda se vincula con la decisión de no beber (estrategias para manejar la situación) y la tercera es utilizar estrategias que les permitan rechazar el consumo (estrategias de control momentáneo).
A partir del análisis de la información obtenida durante la aplicación de los grupos focales, puede concluirse que el abuso de alcohol debe prevenirse a fin de disminuir las consecuencias asociadas al consumo. Una de las formas para hacerlo es a partir del desarrollo de modelos de prevención y atención para segmentos de la población y que son consideradas como poblaciones foco, en las que se ponga énfasis en componentes específicos para disminuir este fenómeno. Los resultados de esta investigación corresponden a la identificación de elementos específicos enfocados a la exposición a la oportunidad y la tentación al consumo, que permitirán el desarrollo de materiales psicoeducativos y alternativas de prevención centradas en habilidades de enfrentamiento a situaciones cotidianas y específicas al consumo de alcohol.

\section{REFERENCIAS}

1. Encuesta Nacional de Adicciones (2008). México: Encuesta Nacional de Adicciones. Consejo Nacional Contra las Adicciones, Instituto Nacional de Psiquiatría Ramón de la Fuente, Dirección General de Epidemiología, Instituto Nacional de Estadística, Geografía e Informática; 2010.

2. Villatoro J, Gutiérrez M, Quiroz N, Moreno M et al. Encuesta de Estudiantes de la Ciudad de México 2006. Prevalencias y Evolución del Consumo. Salud Mental 2009;32(4):287-297.

3. Muss R. Teorías de la adolescencia. México: Paidos; 2004.

4. Lerner RM, Galambos N. Adolescent development challenges and opportunities for research, programs and policies. En: Spence JT (ed.). Annual review o psychology. Palo Alto, CA: 1998.

5. Watson RI, Clay-Lindgren H. Psicología del niño y del adolescente. Primera edición. México: Limusa; 1991.

6. Fountain G. Adolescent into adult: An inquiri. J Am Psychoanal Assoc 1961:9:417-432.

7. Kandel DB, Logan JA. Patterns of drug use from adolescence to young adulthood: I Periods of risk for initiation, continued use and discontinuation. Am J Public Health 1984:74:660-666.

8. Mayers MG, Brown SA, Tate $S$ et al. Toward brief intervention for adolescents with substance abuse prevention of comorbid psychiatric problems. En: Monti PM, Colby SM, O’Leary TA (eds.). Adolescents, alcohol, and substance abuse. New York: Gilford PressPublications; 2001.

9. Spear L. The adolescent brain and the college drinker: Biological basis of propensity to use and misuse alcohol. J Stud Alcohol 2002;(supl)14:71-81.

10. Zuckerman M. Behavioral expressions and biosocial bases of sensation seeking. Cambridge: Cambridge University Press;1994.

11. Villatoro J, Medina-Mora ME, Cardiel H, Fleiz C et al. Consumo de drogas, alcohol y tabaco en estudiantes del Distrito Federal: medición otoño 1997. Reporte Global del Distrito Federal. México: SEP, IMP; 1999.

12. Gardner SE, Brounstein PJ, Stone DB. Science-based substance abuse prevention: A guide. Rockville, MD: Department of Health and Human Service; 2001.

13. Solis L, Gómez P, Ponce M. Modelo de prevención en Centros de Integración Juvenil. En: CONADIC (ed.). Serie planeación: Modelos preventivos. México: CONADIC; 2003.

14. Wagner F, González-Forteza C, Aguilera RM, Ramos-Lira L et al. Oportunidades de exposición al uso de drogas entre estudiantes de secundaria de la Ciudad de México. Salud Mental 2003:2(2):22-32.

15. Winters KC. Assessing adolescent substance use problems and others areas of functioning: State of art. En: Monti PM, Colby SM, O'Leary TA (eds.). Adolescents, alcohol, and substance abuse. New York: Gilford PressPublications; 2001.

16. Wills TA, Shiffman S. Coping and substance use framework. En: Shi- 
ffman S, Wills TA (ed.). Coping an substance use. Orlando, FL: Academic Press; 1985.

17. Mayers M, Brown S, Mott M. Coping as a predictor of adolescent substance abuse treatment outcome. J Subst Abuse 1993;5(1):15-29.

18. Wagner E, Myers M, Mcinich J. Stress-coping and temptation-coping as predictors of adolescent substance use. Addict Behav 1999;24(6):769-779.

19. Reboussin BA, Anthony JC. Latent class marginal regression models for modeling youthful drug involvemet and its suspected influences. Stad Med 2001;20(4):623-629.

20. Wagner FA, Anthony JC. From first drug use to drug dependence; developmental periods of risk for dependence upon marijuana, cocaine, and alcohol. Neuropsychopharmacology 2002;26(4):479-488.

21. Monti PM, Rohsenow DJ, Rubonis AV, Niaura RS et al. Cue exposure with coping skills treatment for male alcoholics a preliminary investigation. J Consult Clin Psychol 1993;61(6):1011-1019.

22. Lazaruz RS. The psychology of coping: Issues of research and assessment. En: Coehlo GV, Hamburg DA, Adams JE (eds.). Coping and adaptation. New York: Basic Book; 1974.

23. Wills TA, Hirky AE. Coping and substance use: A theoretical model and review of the evidence. En: Zeidner M, Endler N (eds.). Handbo- ok of coping: Theory, research, applications. New York: Wiley; 1996; pp279-302.

24. Vaughn S, Shay-Schumm J, Sinagub J. Focus group interviews in education and psychology. California: Sage Publications; 1996.

25. Mariño MC, González-Forteza GC, Andrade P, Medina-Mora ME. Validación de un cuestionario para detectar adolescentes con problemas por uso de drogas. Salud Mental 1998;1:27-36.

26. Bandura A. Social foundation of thought and action. New York: Prentice Hall; 1985.

27. Cellucci T. The prevention of alcohol problems: conceptual and methodological issues. En: Miller PM, Nirenberg TD (eds.). Prevention of alcohol abuse. New York: Plenum Press; 1982; pp15-33.

28. Zucker RA. Developmental aspects of drinking through the young adult years. En: Blane HT, Chafetz ME (eds.). Youth, alcohol and social policy. New York: Plenum; 1979.

29. Plant MA. Learning to drink. En: Grant M, Gwinner P (eds.). Alcoholism in perspective. Baltimore:University Park Press; 1979.

30. Biddle BJ, Bank BJ, Malin MM. Social determinants of adolescent drinking: what they think, what they do and what I think they do. J Studies Alcohol 1980;41:215-241.

Artículo sin conflicto de intereses 